10 years ESJ

Special edition

\title{
El Comportamiento del Consumidor Baby Boom en Época del COVID-19 un Estudio en México, Colombia y Ecuador
}

\author{
Laura Fischer \\ Universidad Nacional Autónoma de México \\ Emigdio Larios-Gómez \\ Benemérita Universidad Autónoma de Puebla, México \\ Diana López. \\ Universidad Jorge Tadeo Bogotá, Colombia \\ Mayra Ortega-Vivanco \\ Universidad Técnica Particular de Loja, Ecuador
}

Doi:10.19044/esj.2021.v17n4p28

Submitted: 28 October 2020

Accepted: 07 December 2020

Published: 07 February 2021
Copyright 2021 Author(s)

Under Creative Commons BY-NC-ND

4.0 OPEN ACCESS

Cite As:

Fischer L., Larios-Gómez E., López D. \& Ortega-Vivanco M (2021). El Comportamiento del Consumidor Baby Boom en Época del COVID-19 un Estudio en México, Colombia y Ecuador. European Scientific Journal, ESJ, 17(4), 28.

https://doi.org/10.19044/esj.2021.v17n4p28

\section{Resumen}

La generación baby boomers (nacidos entre 1946 y 1964) es la generación que se ha visto más afectada por el virus SARS-CoV-2 o COVID19, por el número de muertes que ha cobrado esta pandemia a nivel mundial, el confinamiento que los países han decretado por esta emergencia sanitaria, se ha visto reflejado en los cambios en el consumo en especial en este segmento de la población. El objetivo de este trabajo de investigación es conocer los cambios en el consumo de las personas de esta generación en México, Ecuador y Colombia por la situación de aislamiento producto del COVID-19, Se realizó un estudio cuantitativo a una muestra de 376 personas. Los resultados muestran que los baby boomers ha cambiado sus hábitos de comportamiento en especial en la forma de socializar con su familia y amigos, teniendo que aprender nuevas tecnologías en línea, así mismo para la compra de sus alimentos, ya que esta generación ha tenido que adaptarse al uso de compran en línea. No encontrando diferencias significativas entre hombres y mujeres en este tipo de comportamiento. 
Palabras clave: Baby Boomers, Consumo Generacional, Consumo En Crisis, Consumo En COVID-19, Nuevos Consumidores De Tecnología

\title{
The Behavior of the Baby Boom Consumer in the Time of COVID-19: A Study in Mexico, Colombia and Ecuador
}

\author{
Laura Fischer \\ Universidad Nacional Autónoma de México \\ Emigdio Larios-Gómez \\ Benemérita Universidad Autónoma de Puebla, México \\ Diana López \\ Universidad Jorge Tadeo Bogotá, Colombia \\ Mayra Ortega-Vivanco \\ Universidad Técnica Particular de Loja, Ecuador
}

\begin{abstract}
Baby boomers have been the most affected by COVID-19 due to the number of deaths worldwide. This study focuses on knowing the changes in their consumption due to confinement as a result of the pandemic in Mexico, Ecuador, and Colombia. A quantitative study was carried out using a sample of 376 people. The results show that they had to learn and adapt to new digital technologies to socialize with their family and friends and to buy their food. No significant differences were found between men and women in this type of behavior.
\end{abstract}

Keyword: Baby Boomers, Generational Consumption, Consumption in Crisis, Consumption in COVID-19, New Technology Consumers

\section{Introducción}

La generación de los baby boomers, son un grupo de personas nacidas entre 1946 y 1964, esta generación se caracteriza por los altos índices de natalidad; en estos años a nivel mundial se desarrollaron cambios que marcaron sus vidas, entre los que se destacan revolución en los medios de comunicación por los avances tecnológicos, la liberación femenina, tanto hombres como mujeres salen a trabajar, el ingreso económico es doble, se reduce notablemente el número de hijos, se reparten labores del hogar, en pocas palabras con esta generación se produjo un boom, (Soto, Bonilla, De León, \& Nuñez 2018)

Ramos \& Mercé (2016), consideran que esta generación ha tenido un mayor nivel de supervivencia, salud, condiciones de vida y logros a nivel educativo, tienen una mejor capacidad para organizar sus acciones, en 
relación a la salud, al dinero que gastan, toman decisiones de forma independiente y participan activamente de la vida social, pero también es la que se ha visto más afectada por la emergencia sanitaria a consecuencia de la epidemia del COVID-19,

Este trabajo de investigación está integrado por varios apartados, en el primero se hace la revisión literaria en la que se aborda las características distintivas y perfil de los Baby boomers, la situación del COVID-19 en el mundo y en específico en México, Colombia y Ecuador, así como se muestran algunos resultados de estudios sobre consumo, comportamiento y hábitos de compra de esta generación previos a esta contingencia sanitaria, El siguiente apartado se presenta el objetivo, metodología empleada, y resultados obtenidos, y finalmente se muestran las conclusiones del trabajo.

\section{Revisión literaria}

La clasificación generacional se basa en el periodo de nacimiento de un grupo de personas, las cuales están marcadas por haber vivido acontecimientos similares, tomando en cuenta aspectos como: guerras, cambios tecnológicos, enfermedades y situaciones específicas de una sociedad. (Soto, et al., 2018). De acuerdo con Mcrindle \& Wolfinger (2010) una generación es un grupo de personas nacidas en la misma época, desarrolladas en un mismo tiempo, semejantes en edad, condiciones, preferencias, valores, motivaciones, formas de trabajo, eventos y experiencias similares. Cada generación cuenta con variables socioeconómicas y geográficas específicas, encontrando que cada generación tiene características diferentes (Rivera, 2009; Mosco, 2012), una de estas generaciones es la llamada baby boom.

Se considera baby boom a la generación en donde se reporta el mayor número de nacimientos de todos los tiempos, comprende a las personas nacidas entre 1946 y 1964 (Bonvalet, Clément \& Ogg, 2014); se le conoce también como: "Generación del auge de la natalidad" o "Generación de la postguerra" Grace (2001), el fenómeno del "baby boom" en los países industrializados fue un proceso demográfico contradictorio en la lógica de una fecundidad en declive según la transición demográfica. Van Bavel \& Reher (2013) explican que este fue

“... un período de resurgimiento demográfico en países desarrollados, especialmente aquellos que participaron en la II Guerra Mundial, que ocurrió entre mediados y finales de la década de 1940s, y la última parte de los sesentas o inicios de los setentas. La dinámica de la reproducción cambió durante este período, con tasas de matrimonio acelerándose, fecundidad total aumentando, y el número de nacimientos incrementándose sustancialmente..." (p.257). 
En América Latina se ha estudiado poco el fenómeno del "baby boom" porque la mayoría de los países se encontraban en condiciones de alta natalidad, por lo que la investigación se enfocaba en el inicio del descenso. No obstante, algunos autores han notado brevemente incrementos temporales en la natalidad a mediados del siglo XX: (Zavala de Cosío 1992; Pérez Brignoli 2010). La evidencia señala, que el descenso de la natalidad comenzó a darse durante la segunda mitad del siglo XX (excepto en países, como Argentina, Cuba y Uruguay), en donde existían niveles superiores a los seis hijos por mujer, y empezó a descender esta natalidad a partir de la adopción de métodos de planificación familiar. (Chackiel \& Schkolnik, 2003; Zavala de Cosío, 1992; Brenes-Camacho, 2014).

Esta generación de acuerdo a Ramos \& Mercé, (2016), se caracteriza por la gran cantidad de cambios políticos, sociales, tecnológicos, musicales, entre los que destacan el movimiento feminista, la independencia y preparación educativa de la mujer y su inserción al mercado laboral, ruptura con la moral religiosa, la autodeterminación, el consumismo, la autoexpresión, En relación a la estructura familiar, se reduce el gran número de hijos, el divorcio no está mal visto (Fingerman, Pillemer, Silverstein \& Suitor, 2012);

Debido al periodo de tiempo de edad tan grande que comprende esta generación, existen dos categorías de baby boomers, el primero denominado Leading-Edge que va de 1946 a 1955 y los segundos Jonesers de 1956 a 1964 (Green, 2003) Los Leading-Edge ya están jubilados, no tienen padres, sus hijos están fuera de casa, planean su vida junto a su pareja deseando viajar, compartir, descubrir nuevos lugares, hacer todo lo que no pudieron por trabajar y sobre todo descansar de la larga vida laboral a la que dedicaron muchos años. (Angulo 2016) En cambio los Jonesers se encuentran en su plenitud profesional, tienen hijos que todavía dependen económicamente de ellos, por lo que sus necesidades giran alrededor del bienestar familiar que les exige seguir activos en el trabajo (Angulo, 2016)

Actualmente esta generación la constituyen personas que conscientes de su envejecimiento; cuidan de su salud, de su economía, son optimistas, se ejercitan, tienen gran deseo por vivir, son capaces de tomar decisiones propias de forma independiente y de llevar una vida social activa (Soto, et al 2018; Blein, Lavoie, Guberman \& Olazabal, 2009).

En relación al comportamiento de compra de esta generación, se muestran diferentes investigaciones: algunos resultados de la investigación "Boomers shoppers today and tomorrow: Following the Money." realizada por Unilever (2007): mostrando que: Invierten más dinero, les agrada la calidad y no importa el tiempo que tarden al hacer sus compras; se guían por recomendaciones, desean ser bien atendidos, hayan las ofertas atractivas y comprar se traduce en diversión, es invertir tiempo en lo que les atrae y 
desean sentir que hicieron compras inteligentes; es decir, excelente precio en relación a la calidad del producto adquirido

En The Nielsen Global Generational Lifestyles Survey en 2015 (Nielsen 2015), encontraron que el 29\% de los Baby Boomers comen fuera de casa al menos una vez a la semana; llevar un estilo de vida saludable es muy importante para ellos, en su vocabulario se encuentran atributos como bajo en sodio" o "bajo en azúcar", el 67\% están dispuestos a pagar por alimentos con estas características, el $74 \%$ se encuentra satisfecho con su ocupación laboral, 65\% tiene buena relación con sus colegas, $65 \%$ considera que su lugar de trabajo es agradable y $62 \%$ se siente cómodo en su trabajo y vida cotidiana; por otro lado, existe insatisfacción del $47 \%$ respecto a sus ingresos, del $44 \%$ en cuanto beneficios médicos, $35 \%$ de su plan de retiro (pensión) y $29 \%$ en prestaciones de plan de ahorro. Los Baby Boomers se informan a través de fuentes tradicionales como TV, medios impresos y radio; a un $60 \%$ le interesa hacer ejercicio y estar saludable; $44 \%$ dedica tiempo a su familia; sólo al $21 \%$ le interesa seguir ganando dinero, Los hombres constituían la fuerza laboral y la mujer eran la figura del consumo al interior del hogar.(Nielsen 2015).

Salesforce (2016) realizó un estudio para identificar cuáles son las preferencias de los consumidores a la hora de investigar, comprar y comunicarse con marcas de consumo, el resultado muestra que el $63 \%$ de los Baby Boomers se decide más por comprar el producto en una tienda física, $26 \%$ pertenece a algún programa de lealtad de marca, el 33\% hablan bien o se quejan de alguna marca o producto en público y $22 \%$ comparte información personal con una marca a cambio de recibir servicios y descuentos personalizados.

Y el estudio realizado en Colombia por Lerma \& Paredes, (2015), donde encontraron que los baby boomers son una fuerza laboral estable, trabajaban y ahorraban para adquirir bienes para su familia, principalmente inmuebles y electrodomésticos, gastan más en vacaciones, reformas de hogar, carros, servicios financieros e inmuebles que otras generaciones. La televisión los educó como consumidores, influenció en su comportamiento de consumo hacia artículos para el hogar y también les transmitió valores tradicionales, al mismo tiempo que los informaba sobre acontecimientos internacionales. Son consumidores de medios masivos tradicionales: la televisión, la radio y la prensa. Les gusta mantener informados acerca de su país y el mundo, Son idealistas y optimistas.

$\mathrm{Su}$ consumo lo relacionaban con la acumulación de bienes que significaba el progreso de su familia y aseguraba un futuro mejor para sus hijos. Es una generación preocupada por las características funcionales de los productos. (Arbeláez \& Franco, 2013). El consumo y uso de sus objetos simbólicos de consumo, permitían compartir tiempo en familia y mostrar 
socialmente el estatus de poseerlos, del progreso económico y el valor del ahorro. (Lerma, et al., 2015)

En un estudio realizado en México por Soto et al., (2018) encontraron que: las mujeres son las que más cuidan de su salud, se preocupan por el bienestar financiero de su familia y les interesa brindar apoyo a su comunidad. IAB México en su estudio de Audio digital en México (2018), encontró que el $40 \%$ de los Baby Boomers prefieren las plataformas tradicionales para sus actividades de consumo, el 99\% declaró escuchar música y el $17 \%$ lo hace a través de la radio convencional y en relación a la compra de productos en el estudio realizado por Barbery, Pastor, \& Sempértegui, (2018) encontraron que esta generación tienen miedo de realizar compras online por el temor que sea una estafa, que el producto no llegue a su destino final o llegue averiado y que les roben la información de su tarjeta de crédito.

En un estudio llevado a cabo en Ecuador por Dávila, M, (2012) se encontró que los Baby Boomers tienen dificultad en acoplarse con personas de menor edad, siguen pautas de conducta ya establecidas, renuencia para aceptar los cambios; la comunicación personal es la más utilizada se conserva el nivel de fiabilidad y credibilidad por medio de la palabra para el desarrollo de las actividades laborales, reforzando de esta manera la dinámica cotidiana de las interacciones individuales. Así mismo en Ecuador, Maldonado (2016), muestra en su estudio que los Baby Boomers tienen una relación escaza o casi nula con la publicidad y las marcas que cuentan con sitios oficiales en línea y están interesados en temas como servicios gubernamentales, salud, agencias de viajes, educación, enciclopedias y bolsas de trabajo.

La vida para este grupo de edad, se desarrollaba normalmente, hasta que en el contexto mundial aparece el Coronavirus, COVID-19, a finales de diciembre de 2019, con el brote de neumonía de etiología desconocida -en ese entonces- en Wuhan, provincia de Hubei en China. El Centro Chino para el Control y la Prevención de Enfermedades (CCDC) lo identificó como un nuevo beta-coronavirus (2019-nCoV), ahora conocido oficialmente como Coronavirus 2 del síndrome respiratorio agudo severo [SARS-CoV-2] (Gorbalenya, 2020), el cual se extendió rápidamente por todo el mundo, siguiendo una curva de crecimiento exponencial, que fue clasificada como una pandemia por la Organización Mundial de la Salud (OMS) el 11 de marzo de 2020, en el periodo (06/04/2020 17:00 GMT) en el análisis mundial del contagio del COVID-19 en 209 países, los países con más contagios eran EE.UU. con 290,024 casos, seguido de España con 124,736, Italia con 124,632, Alemania con 92,150, China con 82,543, Francia con 82,165 , Irán con 55,743 casos, Reino Unido, 41,903, Turquía con 23,934 y Suiza con 20,278 casos. (WHO, 2020). Específicamente en América Latina, 
que hasta el momento suma más de 706,798 casos confirmados y 38,864 muertes. (24 de mayo de 2020) encontrándose en pleno pico de la pandemia por el coronavirus, (businessinsider.mx, 2020) esta crisis sanitaria constituye un desafío que amenaza la estabilidad social y económica mundial

Las personas de más de 60 años son el grupo más vulnerable, en donde se han registrado el mayor número de muertes por COVID-19. Todos los países para que no se propagara el contagio y se desbordaran los servicios sanitarios adoptaron la medida del confinamiento en sus hogares, siendo este grupo de edad el que fue más limitado en sus actividades, hasta evitando que saliera a la compra de sus alimentos y medicinas, esta medida todavía al 20 de junio de 2020 se ha seguido manteniendo en varios países para este grupo de edad

Este confinamiento reportado por especialistas en salud mental, está teniendo reacciones y estados psicológicos observados en la población (Torales, O'Higgins, Castaldelli-Maia \& Ventriglio, 2020). Así, Shigemura, Ursano, Morganstein, Kurosawa \& Benedek, (2020) informan que las primeras respuestas emocionales de las personas incluyen miedo e incertidumbre extremos. Por otra parte, y dado el miedo y percepciones distorsionadas de riesgo y consecuencias dañinas informando de comportamientos sociales negativos. Estos estados y conductas pueden dar pie a problemas en salud mental publica incluidas reacciones de angustia (insomnio, ira, miedo extremo a la enfermedad incluso en personas no expuestas), y de comportamientos riesgosos para la salud como mayor uso de alcohol y tabaco y aislamiento social), trastornos específicos como trastorno de estrés postraumático, trastornos de ansiedad, depresión, y somatizaciones. En el estudio realizado en china por (Wang, Pan, Wan \& Xu, 2020), encontraron que la información específica y actualizada sobre la salud (p. Ej., tratamiento, situación de brote local y medidas de precaución específicas como higiene de manos y uso de una mascarilla) se asociaron con un menor impacto psicológico del brote y menores niveles de estrés, ansiedad, y depresión

Los Baby boom han tenido que cambiar su comportamiento en respuesta al confinamiento al que se han visto sometidos, estos cambios se pueden evidenciar en los consumos cotidianos de productos, servicios y actividades que usualmente realizaban, Conocer esos cambios son importantes, debido a que han tenido que enfrentarse a cambios psicológicos, tecnológicos, culturales, entre otros, que los pueden impactar en un futuro inmediato en nuevos hábitos de comportamiento y consumo.

\section{Método}

La investigación tiene como objetivo conocer los cambios en el consumo de esta generación entre hombres y mujeres a causa del 
confinamiento por esta pandemia en México, Ecuador y Colombia. Se realizó una investigación cuantitativa, con medición transversal. Se calculó la muestra; aplicando un nivel de confianza del 95\% y un margen de error del 5.2\%, obteniéndose un tamaño de muestra de 376 personas de México, Ecuador y Colombia, Se diseñó un cuestionario con 26 ítems, conformado de preguntas categóricas y escalade Likert.

Se validó el instrumento obteniendo un Alfa de Cronbach de 0.807 lo cual indica que es válido y fiable de acuerdo al criterio general de George y Mallery (2013); se aplicó a personas de un rango de edad que va de los 51 a los 71 años el trabajo de campo se realizó on line con el Survey monkey, del 29 de mayo al 8 de junio de 2020, periodo en que las familias se encontraban en confinamiento en México, Ecuador y Colombia. El análisis de información se hizo mediante el paquete estadístico SPSS versión 21. Los constructos estudiados fueron: Constructo 1. Factores personales (consumo masivo de entretenimiento), 2. Factores sociales (Comportamientos sociales en tiempo de distanciamiento, relaciones sociales) 3. Factores Psicológicos (Ejercicio y salud mental), 4. Factores culturales, 5. Comportamiento en el consumo

El perfil de las personas que integran la muestra de esta investigación tienen las siguientes características: del total de la muestra el $57.2 \%$ son mujeres, y el resto hombres. En cuanto a su estado civil, fueron: $64.4 \%$ casado, $14.4 \%$ soltero, $9 \%$ divorciado con hijos en casa, $4.5 \%$ divorciado sin hijos en casa $4 \%$ unión libre, $2.4 \%$ viudo con hijos en casa y $1.3 \%$ viudo sin hijos en casa. En relación al nivel educativo el $43.4 \%$ posgrado, $36.7 \%$ licenciatura, $10.1 \%$ preparatoria, $7.7 \%$ carrera técnica, $1.1 \%$ secundaria y $1.1 \%$ primaria. En los cinco constructos analizados se aplicó la Prueba de Mann-Whitney la cual se basa en una comparación de cada observación de una muestra xi con cada observación en la segunda muestra y j, Si las muestras tienen la misma mediana, entonces cada observación tiene un 0,5 (50\%) de oportunidad de ser mayor o menor que la observación correspondiente de la otra muestra. En el caso de la presente investigación se utilizó como variable independiente el sexo. Tomando como: H1. Existen diferencias en el consumo de la generación Baby boom entre hombres y mujeres

\section{Hallazgos}

Constructo 1. Factores personales (consumo masivo de entretenimiento), Se refiere a las actividades que están realizando durante el confinamiento para atenuar su aislamiento de las labores cotidianas, encontrando que no existen diferencias por sexo (Tabla 1). 
Tabla 1. Prueba de Mann-Whitney. Constructo Factores personales (consumo masivo de entretenimiento)

\begin{tabular}{|c|c|c|c|c|c|}
\hline & \multicolumn{1}{|c|}{$\begin{array}{c}\text { U de } \\
\text { Mann- } \\
\text { Whitney }\end{array}$} & $\begin{array}{c}\text { W de } \\
\text { Wilcoxon }\end{array}$ & Z & $\begin{array}{c}\text { Sig. } \\
\text { Asintótica } \\
\text { (bilateral) }\end{array}$ \\
\hline 1.1 & $\begin{array}{c}\text { He consumido programas y películas a } \\
\text { través de las plataformas como Netflix }\end{array}$ & 16209.500 & 29250.500 & -1.095 & 0.274 \\
\hline 1.2 & $\begin{array}{c}\text { He aumentado mi interés en las } \\
\text { plataformas de streaming asociados a } \\
\text { videojuegos y música }\end{array}$ & 16830.500 & 40050.500 & -0.470 & 0.638 \\
\hline 1.3 & $\begin{array}{c}\text { Escucho más la radio que antes del inicio } \\
\text { del brote del COVID-19 }\end{array}$ & 16878.500 & 29919.500 & -0.423 & 0.673 \\
\hline 1.4 & $\begin{array}{c}\text { La cocina es una de las actividades que } \\
\text { he incrementado desde el brote del } \\
\text { COVID-19 }\end{array}$ & 12539.500 & 25580.500 & -4.732 & 0.000 \\
\hline 1.5 & $\begin{array}{c}\text { He incrementado la socialización con mi } \\
\text { familia a través de la comunicación y } \\
\text { pasatiempos }\end{array}$ & 16827.500 & 29868.500 & -0.487 & 0.626 \\
\hline & \multicolumn{3}{|c|}{ a. Variable de agrupación: sexo } & & \\
\hline
\end{tabular}

Esta tabla muestra la Prueba U de Mann-Whitney, utilizando como variable de agrupación el género. En esta tabla se identifica que de los 5 ítems que integran el constructo, solo uno de ellos tienen una significancia $<0.05$ La cocina es una de las actividades que he incrementado desde el brote del COVID-19, actividad que en este grupo de edad, ha sido preponderante de la mujer lo que indica que en este constructo no existen diferencia significativa entre hombres y mujeres a un $* p<0.05$.

Encontramos que en relación a "He aumentado mi interés en las plataformas de streaming asociados a videojuegos y música" y "Escucho más la radio que antes del inicio del brote del COVID-19," tuvieron una media de 2.58 y 2.61 , respectivamente estando en los rangos de desacuerdo, los otros tres ítems se encuentran en los valores favorables encontrando un incremento en estas actividades.

Constructo 2. Factores sociales (Comportamientos sociales en tiempo de distanciamiento, relaciones sociales). Se refiere a las actividades tecnológicas de socialización que están realizando este grupo de personas en la situación de aislamiento (Tabla 2).

Tabla 2. Prueba de Mann-Whitney. Constructo Factores sociales (Comportamientos sociales en tiempo de distanciamiento, relaciones sociales

\begin{tabular}{|c|c|c|c|c|c|}
\hline & & $\begin{array}{c}\text { U de } \\
\text { Mann- } \\
\text { Whitney }\end{array}$ & $\begin{array}{c}\text { W de } \\
\text { Wilcoxon }\end{array}$ & Z & $\begin{array}{c}\text { Sig. Asintótica } \\
\text { (bilateral) }\end{array}$ \\
\hline $2 . .1$ & $\begin{array}{c}\text { La comunicación online y las } \\
\text { redes sociales han sido utilizadas } \\
\text { como plataformas para reconectar } \\
\text { con el entorno cercano durante } \\
\text { esta crisis }\end{array}$ & 16783.500 & 40003.500 & -0.551 & 0.581 \\
\hline 2.2 & $\begin{array}{c}\text { He utilizado la comunicación } \\
\text { online y las redes sociales como }\end{array}$ & 16442.000 & 39662.000 & -0.902 & 0.367 \\
\hline
\end{tabular}




\begin{tabular}{|c|c|c|c|c|c|}
\hline & $\begin{array}{c}\text { plataformas para reconectar en el } \\
\text { ámbito profesional durante esta } \\
\text { crisis }\end{array}$ & & & & \\
\hline $2 . .3$ & $\begin{array}{c}\text { Con el confinamiento he } \\
\text { aumentado el uso de las } \\
\text { aplicaciones de mensajería como } \\
\text { WhatsApp }\end{array}$ & 17137.500 & 40357.500 & -0.181 & 0.857 \\
\hline $2 . .4$ & $\begin{array}{c}\text { He utilizado las aplicaciones de } \\
\text { videoconferencia (Zoom, Google, } \\
\text { Classroom) en actividades } \\
\text { recreativas }\end{array}$ & 16982.500 & 30023.500 & -0.329 & 0.742 \\
\hline $2 . .5$ & $\begin{array}{c}\text { Para actividades profesionales y } \\
\text { educativas estoy utilizado las } \\
\text { aplicaciones de videoconferencia } \\
\text { como Zoom, Google o Classroom }\end{array}$ & 16660.500 & 39880.500 & -0.668 & 0.504 \\
\hline & \multicolumn{2}{|c|}{ a. Variable de agrupación: Sexo } & \\
\hline
\end{tabular}

Esta tabla muestra la Prueba U de Mann-Whitney, utilizando como variable de agrupación el género. En esta tabla se identifica que de los 5 ítems que integran el constructo, ninguno de ellos tienen una significancia $<0.05$ lo que indica que en este constructo no existen diferencia significativa entre hombres y mujeres a un $* p<0.05$.

En relación a la forma de socializar utilizando todas las herramientas existentes de tecnología, se aprecia un aumento encontrado en los cinco ítems de este constructo superiores a la media de 4.2, equivalente a de acuerdo resultado que difiere del estudio realizado por (Nielsen, 2015) en donde un $86 \%$ utiliza los medios tradicionales como TV, medios impresos y radio para le interesa hacer ejercicio y estar saludable; así como que las mujeres eran la figura del consumo al interior del hogar (Nielsen, 2015), las personas de este grupo han tenido que adaptarse al confinamiento, recurriendo a la tecnología para socializar con los amigos y familiares

Constructo 3. Factores Psicológicos (Ejercicio y salud mental), Se refiere a las actividades que están realizando los baby bommers para mantenerse física y mentalmente sanos en medio de este confinamiento producto de la emergencia sanitaria (Tabla 3 ).

Tabla 3. Prueba de Mann-Whitney. Constructo Factores Psicológicos

(Ejercicio y salud mental)

\begin{tabular}{|c|c|c|c|c|c|}
\hline & & $\begin{array}{c}\text { U de } \\
\text { Mann- } \\
\text { Whitney }\end{array}$ & $\begin{array}{c}\text { W de } \\
\text { Wilcoxon }\end{array}$ & Z & $\begin{array}{c}\text { Sig. } \\
\text { Asintótica } \\
\text { (bilateral) }\end{array}$ \\
\hline 3.1 & $\begin{array}{c}\text { Estoy realizado ejercicios de } \\
\text { equilibrio cuerpo y mente en la } \\
\text { actividad cotidiana durante el } \\
\text { COVID 19 }\end{array}$ & 17074.000 & 30115.000 & -0.233 & 0.816 \\
\hline 3.2 & $\begin{array}{c}\text { El humor ha sido uno de los } \\
\text { medios escogidos para mantener } \\
\text { el ánimo en tiempos de } \\
\text { confinamiento }\end{array}$ & 17165.500 & 40385.500 & -0.145 & 0.884 \\
\hline
\end{tabular}




\begin{tabular}{|c|c|c|c|c|c|}
\hline 3.3 & $\begin{array}{c}\text { He comprado seguros de salud } \\
\text { privados para mejorar la } \\
\text { atención médica }\end{array}$ & 17190.500 & 40410.500 & -0.117 & 0.906 \\
\hline 3.4 & $\begin{array}{c}\text { Me encuentro optimista sobre el } \\
\text { desarrollo y la evolución de la } \\
\text { pandemia COVID-19 }\end{array}$ & 16433.000 & 39653.000 & -0.864 & 0.388 \\
\hline
\end{tabular}

$$
\text { a. Variable de agrupación: Sexo }
$$

Esta tabla muestra la Prueba U de Mann-Whitney, utilizando como variable de agrupación el género. En esta tabla se identifica que de los 4 ítems que integran el constructo, ninguno de ellos tienen una significancia $<0.05$ lo que indica que en este constructo no existen diferencia significativa entre hombres y mujeres a un $* p<0.05$.

En relación a la media encontramos que ha excepción de "He comprado seguros de salud privados para mejorar la atención médica" que tuvo un valor de 2.15 , equivalente a desacuerdo, los otros tres ítems fueron indiferentes (ni de acuerdo, ni en desacuerdo), este resultado refleja que este grupo de edad, ya tiene la seguridad médica, producto de su pensión por jubilación. En relación con la pregunta "Estoy realizado ejercicios de equilibrio cuerpo y mente en la actividad cotidiana durante el COVID-19", En el estudio de Nielsen (2015) en donde el 60\% contestó que realizaba ejercicios, en este rubro, los encuestados tuvieron como media 3.67, valor que coincide con los resultados del estudio mencionado

Constructo 4. Factores culturales, Se refiere a las costumbres que realizaban cotidianamente este grupo de personas en relación a su alimentación (Tabla 4).

Tabla 4. Prueba de Mann-Whitney. Constructo Factores culturales

\begin{tabular}{|c|c|c|c|c|c|}
\hline & & $\begin{array}{c}\text { U de } \\
\text { Mann- } \\
\text { Whitney }\end{array}$ & $\begin{array}{c}\text { W de } \\
\text { Wilcoxon }\end{array}$ & $\mathbf{Z}$ & $\begin{array}{c}\text { Sig. } \\
\text { Asintótica } \\
\text { (bilateral) }\end{array}$ \\
\hline 4.1 & $\begin{array}{c}\text { He cambiado mis costumbres } \\
\text { en la alimentación por el } \\
\text { COVID-19 }\end{array}$ & 16691.000 & 29732.000 & -0.616 & 0.538 \\
\hline 4.2 & $\begin{array}{c}\text { He incrementado la preferencia } \\
\text { por el consumo de productos } \\
\text { locales }\end{array}$ & 14449.500 & 27490.500 & -2.887 & 0.004 \\
\hline
\end{tabular}

a. Variable de agrupación: Sexo

Esta tabla muestra la Prueba U de Mann-Whitney, utilizando como variable de agrupación el género. En esta tabla se identifica que de los 2 ítems que integran el constructo, el ítem

"He incrementado la preferencia por el consumo de productos locales "tienen una significancia $<0.05$ lo que indica que existen diferencia significativa entre hombres y mujeres a un $* p<0.05$.

Esta generación como lo refieren los estudios de Nielsen (2015), Lerma (2015) están conscientes de su salud y consumo de los productos sanos, característica que se ve reflejada en las respuestas obtenidas en estos dos ítems, ambos respuestas con valores de 3.55 y 3.88, respectivamente; 
valores que representan una respuesta indiferente, por lo que encontramos que en la situación del aislamiento producto del COVID-19, no modificaron sus hábitos alimenticios.

En el Constructo 5: Comportamiento en el consumo, se refiere a la forma como adquieren los productos (Tabla 5).

Tabla 5. Prueba de Mann-Whitney. Constructo Comportamiento en el consumo

\begin{tabular}{|c|c|c|c|c|c|}
\hline & N & $\begin{array}{c}\text { U de } \\
\text { Mann- } \\
\text { Whitney }\end{array}$ & $\begin{array}{c}\text { W de } \\
\text { Wilcoxon }\end{array}$ & Z & $\begin{array}{c}\text { Sig. } \\
\text { Asintótica } \\
\text { (bilateral) }\end{array}$ \\
\hline 5.1 & $\begin{array}{c}\text { He aumentado los } \\
\text { servicios web de } \\
\text { compra de alimentos a } \\
\text { domicilio con la crisis } \\
\text { del COVID-19 }\end{array}$ & 16951.000 & 29992.000 & -0.350 & 0.727 \\
\hline 5.2 & $\begin{array}{c}\text { Considero que los } \\
\text { productos básicos } \\
\text { deben ofrecerse a } \\
\text { través de internet }\end{array}$ & 16447.500 & 39667.500 & -0.856 & 0.392 \\
$\begin{array}{c}\text { He aumentado las } \\
\text { compras de productos } \\
\text { que cubren } \\
\text { necesidades básicas } \\
\text { como: alimentación } \\
\text { salud, cuidado } \\
\text { personal en tiempos de } \\
\text { COVID-19 }\end{array}$ & 17182.000 & 40402.000 & -0.125 & 0.901 \\
\hline 5.4 & $\begin{array}{c}\text { He cambiado mi } \\
\text { comportamiento de } \\
\text { compras para los } \\
\text { productos suntuarios }\end{array}$ & 16581.500 & 29622.500 & -0.722 & 0.470 \\
\hline
\end{tabular}

a. Variable de agrupación: Sexo

Esta tabla muestra la Prueba $U$ de Mann-Whitney, utilizando como variable de agrupación el género. En esta tabla se identifica que de los 4 ítems que integran el constructo, ninguno de ellos tienen una significancia $<0.05$ lo que indica que en este constructo no existen diferencia significativa entre hombres y mujeres a un $* p<0.05$.

En los resultados de la media de estos ítems se encontró que habían aumentado los servicios web de compra de alimentos a domicilio con la crisis del COVID-19 (4.23 valor referido a "de acuerdo") este resultado muestra que los comportamientos de consumo se han modificado por la situación de aislamiento generada por la emergencia sanitaria ya que como lo se muestra Salesforce en su estudio, el 63\% de los Baby Boomers se decide más por comprar el producto en una tienda física, teniendo que adaptarse a las nuevas situaciones del entorno; en los demás ítems los valores de la media quedaron en los rangos de indiferente "ni de acuerdo, ni desacuerdo" 


\section{Conclusiones}

Esta generación es la que se ha visto más vulnerable en los efectos de letalidad del COVID- 19, lo cual los ha llevado a ser más estrictos en el seguimiento de las medidas que los gobiernos han adoptado para disminuir el contagio, y que se propague con mayor rapidez este virus en cada país.

Esta situación que se vive actualmente va a traer cambios en esta generación como se refleja en los resultados de esta investigación en especial por la migración e incremento en el uso de la tecnología, lo cual implica cambios más allá de sus hábitos básicos, cambios en la forma de la adquisición de productos y servicios al igual que en el medio de su forma de socialización. Los baby boomr les ha sido difícil adaptarse a estos cambios en aislamiento, ya que este segmento ha tenido que aprender a usar la tecnología para la compra de productos en línea y para socializar con sus familiares y amigos, aunque sus hábitos para mantenerse saludables como en lo referente a ejercicio o cambios en la alimentación no se han modificado. Esta situación que se vive actualmente va a traer cambios en esta generación siendo la más vulnerable al contagio y reaccionará como lo fue pronosticado en el estudio realizado por Russell, Zwanka y Cheryl (2020) sobre los cambios de comportamiento del consumidor que serán causados por la pandemia COVID-19 en el que muestra que la compra en línea será una práctica durante y posterior a la pandemia; será posible que la pandemia afecte permanentemente a todos los productos y servicios que involucren "contacto alto" o proximidad cercana con otros. Ellos prevén un futuro sin contacto físico, la eliminación del apretón de manos, la preferencia de aislamiento, el mantenimiento de un círculo de amigos más pequeño e íntimo, una mayor conciencia de la presencia de gérmenes en las áreas públicas, más entretenimiento en casa, menos asistencia a conciertos y deportes, eventos, menos compartir comida, asistencia a restaurantes y cuestionar la necesidad de volar para reunirse por razones personales o comerciales y mayor uso de plataformas en línea, como Zoom, WebEx, Skype, y FaceTime.

Son importantes los hallazgos de esta investigación a nivel de marketing; puesto que permite la adaptación del canal online para esta generación; para la cual es claro que antes del COVID-19 no tenían un contacto muy alto con la tecnología y mucho menos para comprar productos o socialización, y ahora de una forma forzada deben usarlo; lo cual, representa una oportunidad a nivel de mercado, para implementar estrategias de acercamiento de las marcas a través de los canales virtuales apoyados en medios tradicionales, considerando que aún tienen un alto contacto con medios como televisión y radio, es el momento que las marcas trabajen en facilitarles este nuevo estilo de vida ajeno para ellos, y posiblemente con una percepción de agresividad para y falta de consideración al no tener en cuenta 
sus limitantes y necesidades.

En relación a nuestra hipótesis, los resultados de esta investigación no se encontraron diferencias estadísticamente significativas por sexo; lo cual posibilita que se puedan trabajar estrategias de marketing transversales para esta generación, permitiendo acercar el canal virtual con las marca y el consumidor, construyendo en la mente del consumidor un relacionamiento más amigable con proyección a largo plazo.

\section{References:}

1. Angulo, D. (2016). Los subgrupos de los 'Baby Boomers' y sus hábitos. P\&M Junio. Colombia.

2. Blein, L., Lavoie, J., Guberman, N., \& Olazabal, I. (2009). Vieilliront-ils un jour? Les baby-boomers aidants face à leur vieillissement. Lien Social et Politiques, (62), 123-134.

3. Barbery, D., Pastor, B., Idrobo, D., \& Sempértegui, L. (2018). Análisis comparativo generacional del comportamiento de compra online. Revista Espacios, 39(34), 16-25.

4. Bonvalet, C., Clément, C., \& Ogg, J. (2014). Renewing the family: A history of the baby boomers. New York: Springer.

5. Brenes-Camacho, G. Describiendo procesos históricos de "baby boom" durante mediados del siglo $\mathrm{XX}$ en tres naciones latinoamericanas usando estudios de envejecimiento.

6. Business insider (2020). https://businessinsider.mx/america-latina-esun-nuevo-epicentro-de-la-pandemia-de-coronavirus/ extraído 10 de junio de 2020.

7. Chackiel, J. \& Schkolnik, S. (2003). América Latina: Los sectores rezagados en la transición de la fecundidad (No. 42). United Nations Publications.

8. Dávila, M. (2012). Análisis de la Convivencia entre las diferentes generaciones: Baby Boomers, Generación X y Generación Y, en el sector público; caso Secretaria Nacional de Educación Superior, Ciencia, Tecnología e Innovación (SENESCYT). Tesis Universidad Andina Simón Bolívar, Sede Ecuador.

9. Fingerman, K., Pillemer, K., Silverstein, M., \& Suitor, J. (2012). The baby boomers' intergenerational relationships. The Gerontologist, 52(2), 199-209.

10. Gorbalenya, A. E. (2020). Severe acute respiratory syndrome-related coronavirus-The species and its viruses, a statement of the Coronavirus Study Group. BioRxiv.

11. George, D. \& Mallery, P. (2013). SPSS for Windows step by step: A simple guide Longman Publishers. Nairobi, Kenya Debt structure [Electronic Version], 20, 1389. 
12. Grace J. \& Craig, B. (2001). Desarrollo psicológico. 8 ed. México: Pearson educación

13. Green, B. (2003). Marketing to Leading - Edge Baby Boomers. New York. Paramount market publishinGuberman, N., Lavoie, J., Blein, L., \& Olazabal, I. (2012). Baby boom caregivers: Care in the age of individualization. The Gerontologist, 52(2), 210- 218. http://dx.doi.org/10.1093/geront/gnr140

14. LabMexico (2018). https://www.iabmexico.com/estudios/babyboomers-estudio-audio-digital-2017/, extraído el 20 de junio de 2020.

15. Lerma Cruz, C. E. \& Paredes Bedoya, Y. P. (2015). Objetos Simbólicos De Consumo Y Su Relación Con La Construcción De Identidad De Las Generaciones De Consumidores En La Ciudad De Cali-Colombia (Symbolic Objects of Consumption and its Relationship with the Identity Construction of the Consumers Generations in Cali-Colombia). Revista global de negocios, 36(6), 75-88.

16. Maldonado Ibarra, L. C. (2016). Análisis sobre el uso y manejo de la tecnología móvil en las generaciones Baby Boomer y Generación X (Bachelor's thesis, Quito: Universidad de las Américas.

17. Mascó, A. (2012). Entre Generaciones. Buenos Aires: Temas Grupo Editorial.

18. McCrindle, M. \& Wolfinger, E. (2010). The ABC of XYZ: Understanding the Global Generations. Sydney: University of New South Wales Press.

19. Nielsen (2015). Estilos de Vida Generacionales. An uncommon Sense of the Consumer https://www.nielsen.com/content/dam/nielsenglobal/latam/docs/repor ts/2016/EstilosdeVidaGeneracionale s.pdf

20. Parment, A. (2013). Generation Y Vs Baby Boomers: Shopping behavior, buyer involvement, and implications for retailing. Journal of retailing and consumer services, (20), 189-199.

21. Pérez-Brignoli, H. (2010). La población de Costa Rica 1750-2000: una historia experimental. San Pedro de Montes de Oca: EUCR, Universidad de Costa Rica.

22. Ramos, D. \& Mercé, S. (2016). Claves para el análisis de la participación social en los procesos de envejecimiento de la generación baby boom. Psicoperspectivas, 15(2),53, https://scielo.conicyt.cl/scielo.php?pid=S071869242016000200006\& script=sci_arttext\&tlng $=\mathrm{pt}$

23. Rivera, L. (2009). ¿Dónde Están Los Baby Boomers? FORBES México. https://www.forbes.com.mx/donde-estan-los-babyboomers/extraida 5 de junio de 2020 
24. Russell, J., Zwanka, y., \& Cheryl, B. (2020). Generación COVID-19: un marco conceptual de los cambios de comportamiento del consumidor que serán causados por la pandemia COVID-19, Journal of International Consumer Marketing, DOI: 10.1080 / 08961530.2020 .1771646$.

25. Salesforce (2016). Connected consumer goods report Publicado en 16 junio, 2016 por Javier Pérez Cortijo Santesmases, M. (2004). Marketing: Conceptos y estrategias 5a. Edición. Pirámide, extraida el 15 de junio de 2020

26. Soto, J. M. S., Bonilla, A. H., de León, A. D. L. P., \& Núñez, B. A. (2018). Baby boomers una generación puente. Revista Internacional, 11(3), 47-56.

27. Unilever shows retailers how to follow the money: Study provides insights and solutions on how to capture the Nation's most influential consumer group. Mayo/07/2007. Disponible en: http://www.unileverusa.com/mediacenter/pressreleases/2007/Unileve rshowsretailershowtofollowthemone y.aspx, extraída el 15 de junio de 2020

28. Van Bavel, J. \& Reher, D. S. (2013). What we know and what we need to know about the baby boom. Population and Development Review, 39(2): 257-288.

29. WHO- World Health Organization (2020). Coronavirus disease (COVID-19) Situation report - 51 Coronavirus disease 2019 (COVID-19). 11 March $2020 \quad$ (10:00 GMT). https://www.who.int/docs/default-source/coronaviruse/situationreports/20200311-sitrep-51-COVID-19.pdf?sfvrsn=1ba62e57_10

30. Zavala de Cosío, M. E. (1992). Cambios de fecundidad en México y políticas de población. México, El Colegio de México/FCE. 